\title{
Uso da técnica de tubulização para o reparo de lesões do sistema nervoso periférico
}

\author{
Thiago da Silva Freitas ${ }^{1}$, Leandro Pretto Flores $^{2}$ \\ Unidade de Neurocirurgia do Hospital de Base do Distrito Federal (HBDF), Brasilia, DF, Brasil
}

\section{RESUMO}

As primeiras cirurgias de nervos periféricos começaram a ser realizadas há mais de 150 anos e precocemente se observou que as suturas término-terminais, quando feitas sob tensão, resultavam em limitados resultados funcionais. A utilização de algum tipo de tecido interposto entre os cotos do nervo traumatizado foi logo idealizada como alternativa e, atualmente, o uso de enxertos neurais autólogos permanece como o "padrão-ouro" para o tratamento de lesões extensas do sistema nervoso periférico. Porém, a técnica apresenta algumas limitações, que resultam em morbidade para o paciente. Esses fatos motivaram o desenvolvimento de materiais alternativos para servir como condutor de enxertos, dando início às pesquisas das técnicas de tubulização. Os tubos biológicos, como veias ou de veias enriquecidas com músculo esquelético, demonstraram resultados favoráveis tanto em estudos clínicos quanto em experimentais. O uso de materiais sintéticos também foi alvo de avaliação, apresentando resultados animadores com os tubos de silicone e de ácido poliglicólico.

Esta revisão objetiva descrever a evolução histórica e os resultados dos ensaios clínicos e experimentais obtidos com as técnicas de tubulização para o tratamento das lesões traumáticas do sistema nervoso periférico.

\section{PALAVRAS-CHAVE}

Tubulização. Enxertos de nervos. Cirurgia de nervos periféricos.

\begin{abstract}
Tubulization techniques for the repair of peripheral nervous system injuries

Peripheral nerve surgery remotes to more than 150 years ago, and soon it was observed that terminoterminal sutures performed under tension resulted in poor functional outcomes. The use of some kind of graft tissue interposed between the traumatized nerve stumps was developed to solve these situations, but only in the 70's Millesi proved the efficacy of autologous nerves as an ideal tissue for grafting. Nowadays, the use of these grafts is the "gold-standard" treatment to severe injuries with long gaps in peripheral nerves, but the technique has some limitations. These limitations motivated the search for alternative tissues as source to sustain the grafting, beginning the development of researches about the tubulization techniques. Biological tubes, as veins or veins filled with skeletal muscles, demonstrated good results in experimental and clinical studies. In selected cases, the use of syntetic tubes - as silicon and poliglycolic acid - has also demonstrated good results.

This review aims to describe the historical evolution and the results of the studies about the different tubulizations techniques used for reconstruction of the peripheral nerves injuries.
\end{abstract}

\section{KEY WORDS}

Tubulization. Nerve grafts. Peripheral nerve surgery.

\section{Introdução}

A moderna cirurgia de nervos periféricos iniciou-se há mais de 150 anos, quando as primeiras tentativas de tratamento cirúrgico dessas lesões demonstraram alguma recuperação funcional em pacientes selecionados.
Esses resultados iniciais, aliados às descobertas já estabelecidas do início do século XIX por Waller e Ranvier a respeito da possibilidade de crescimento axonal no coto proximal de nervos seccionados, incentivaram o desenvolvimento de novas técnicas para o tratamento dessas enfermidades ${ }^{40}$. 
Inicialmente, a sutura direta dos cotos lesados apresentava-se como a única alternativa disponível para os pacientes com essas lesões. Entretanto, a análise dos resultados clínicos obtidos com o acúmulo de experiência, principalmente durante as duas grandes guerras do século XX, demonstrou que aquelas suturas primárias feitas sob tensão - como no caso de lesões de extremidade com perda de substância - apresentavam recuperação funcional inadequada ${ }^{33}$.

O conceito de "enxertos" aplicado à cirurgia de nervos periféricos foi introduzido no final da década de 1940, com a apresentação dos resultados cirúrgicos obtidos por Seddon ${ }^{40}$, utilizando múltiplos cabos de enxertos autólogos para o tratamento de lesões nervosas extensas. No início dos anos de 1970, os trabalhos desenvolvidos por Millesi e cols. ${ }^{30-32}$ demonstraram que o uso de enxerto de nervos autólogos apresentava melhores resultados, em termos de recuperação de funções motora e sensitiva, do que as suturas primárias confeccionadas sob tensão. Esses trabalhos abriram o caminho para o uso de enxertos no tratamento das lesões de nervos periféricos.

\section{Enxertos neurais autólogos}

Segundo Millesi e cols..$^{31,32}$, a utilidade de um enxerto neural baseia-se no fato de essa estrutura servir como um guia para o crescimento dos brotos axonais do coto proximal até o coto distal. As observações histológicas experimentais desses autores constataram que a efetividade desses enxertos não dependia somente da sua estrutura, mas também da presença e viabilidade das células de Schwann em seu interior. Essas células têm por finalidade a liberação de fatores químicos que facilitam o crescimento dos brotos axonais e que também orientam esses axônios em direção ao coto distal. A sua ausência provocaria a formação de um "neuroma" no enxerto, dificultando o processo de regeneração axonal.

De acordo com esses princípios, foram definidas as condições para a viabilidade de um enxerto neural autólogo: ausência de necrose no seu tecido conjuntivo de sustentação; ausência de fibrose em seu interior; degeneração walleriana em toda a sua extensão, objetivando a liberação dos tubos de endoneuro para o crescimento dos novos axônios provenientes do coto proximal; e presença e viabilidade das células de Schwann nos tubos endoneurais do nervo doador. Também alguns fatores foram identificados como fundamentais para a seleção de um enxerto ideal, tais como: a organização fascicular; o tamanho e o calibre do enxerto, e a sobrevivência e a revascularização do nervo doador. Além disso, o enxerto neural ideal deve infligir um déficit neurológico o mais discreto possível. Com base nesses critérios, os nervos cutâneos superficiais aparecem como a opção mais viável, pois estes, em geral, apresentam uma organização fascicular bem definida, são normalmente longos e o déficit neurológico restringe-se à perda sensitiva em territórios limitados.

Após curto período inicial sem suprimento sangüíneo, a vascularização de um enxerto é restaurada por meio da anastomose espontânea de vasos do leito receptor com vasos do epineuro e perineuro do nervo enxertado. Idealmente, essa nova circulação e a conseqüente nutrição desse enxerto devem desenvolver-se antes da ocorrência do processo isquêmico, especialmente nas suas porções mais centrais. Assim, quanto maiores o diâmetro e a extensão do enxerto, maior o risco de isquemia central e conseqüente formação de fibrose intraneural. Alguns estudos confirmam a ausência de isquemia e necrose dos nervos cutâneos superficiais quando utilizados no reparo de lesões do sistema nervoso periférico ${ }^{1,6,8}$. Tendo como objetivo melhorar a vascularização dos tecidos enxertados, Strange ${ }^{43}$ desenvolveu a técnica do uso de enxertos neurais autólogos extraídos conjuntamente com seus pedículos vasculares. Essa técnica foi utilizada principalmente por Brooks e Terzis $^{5}$, que definiram os possíveis sítios doadores de enxertos neurais vascularizados, bem como o padrão de vascularização ideal para o uso racional desses enxertos. Mesmo com todos os avanços técnicos obtidos com o uso de enxertos pediculados, não existe, hoje, consenso se estes favoreceriam ou não a regeneração axonal de forma mais efetiva do que os enxertos neurais não-vascularizados.

Apesar do grande avanço obtido no tratamento das lesões nervosas com o uso de enxertos neurais autólogos, principalmente pelo fato de não induzirem reação auto-imune, existem algumas desvantagens e limitações no uso desse tipo de técnica. As principais são: o procedimento de retirada do enxerto requer outra intervenção cirúrgica - nova incisão, novo sítio com risco de infecção; a remoção de um nervo sensitivo provoca déficit que pode tornar-se permanente; a quantidade e o tamanho do material são limitados, oferecendo dificuldades em lesões extensas, como no caso de traumas do plexo braquial ${ }^{45}$.

A despeito dessas desvantagens e limitações, o uso dos enxertos neurais autólogos ainda permanece como "padrão-ouro" na reconstrução das lesões de nervos periféricos, quando não é possível a sutura términoterminal dos cotos. Entretanto, na tentativa de contornar as desvantagens anteriormente citadas, alguns pesquisadores procuraram desenvolver um novo modelo de reconstrução. Diversas pesquisas, utilizando diferentes tipos de materiais biológicos e não biológicos, conver- 
giram no desenvolvimento das técnicas de tubulização, também denominadas "em tubulação". Essas técnicas baseiam-se no uso de materiais não-neurais, os quais teriam a função de envolver a sutura neural e/ou de preencher a falha no tecido nervoso lesado, utilizando um tubo com capacidade de unir os dois cotos. Possuem as vantagens de direcionar o crescimento axonal e ainda isolar o meio neural dos tecidos adjacentes, reduzindo a reação inflamatória no local da(s) sutura(s). Inúmeros esforços foram realizados para a identificação de materiais não-neurais com capacidade de tubulização, sejam eles obtidos a partir de materiais biológicos ou de produtos sintéticos.

\section{Tubos biológicos}

As primeiras descrições do uso de materiais biológico para o tratamento de lesões nervosas periféricas foram feitas por Gluck ${ }^{19}$, em 1880. Esse autor utilizou fragmentos de ossos descalcificados na tentativa de reconstrução de nervos em animais de laboratório. Experiência semelhante foi descrita por Vanlair ${ }^{49}$, que observou, em análises histológicas, a presença de fragmentos axonais em um tubo ósseo descalcificado de 30 milímetros de comprimento. Uma década depois, sinais de crescimento axonal foram encontrados por Bungner ${ }^{9}$ em experimento utilizando um segmento de artéria braquial para reparar nervo hipoglosso de cães, obtendo relativo sucesso funcional. Porém, como a técnica incorria em risco de sangramento e possível isquemia tecidual ocasionados pela retirada de um enxerto arterial, o foco das pesquisas foi dirigido ao uso de tubos venosos.

Nas duas décadas seguintes, Forametti ${ }^{16}$ propôs o uso de tubos venosos como ponte no reparo de nervos periféricos em cães, obtendo comprovação histológica de crescimento axonal, além de sinais clínicos de reinervação. $\mathrm{O}$ primeiro relato do uso de veias para reparo de lesões nervosas em humanos foi feito por Wrede $^{55}$, em 1909. Esse autor obteve boa recuperação sensitiva utilizando um tubo venoso de 45 milímetros de extensão em lesão de nervo digital. Muitos estudos se seguiram a esse, com resultados conflitantes, o que gerou dúvidas a respeito da efetividade desse tipo de tubo. Nos anos de 1940, Weiss ${ }^{54}$ revisou os trabalhos até então produzidos a respeito do uso de veias e tubos sintéticos para o reparo de nervos, concluindo que os limitados resultados observados eram responsáveis pelo pouco entusiasmo sobre a técnica. Finalmente, em 1976, a tubulização era sentenciada como "um procedimento de maus resultados" ${ }^{\prime 4}$.
As pesquisas desenvolvidas nas décadas de $1980 \mathrm{e}$ 1990 propiciaram a evolução do conceito mecânico da tubulização: o conhecimento mais claro a respeito dos mecanismos do crescimento axonal, aliado ao reconhecimento da importância da presença de um suporte físico em falhas neurais com perdas extensas de substância, proporcionou o ressurgimento do interesse sobre a técnica. $\mathrm{O}$ conceito de suporte físico à regeneração axonal serviu como preceito básico da tubulização, que visa basicamente à criação de um ambiente propício ao crescimento desses axônios, livre dos efeitos da reação inflamatória dos tecidos circunjacentes. Com isso, o uso de tubos venosos autólogos foi reassumido nos anos de 1980. Chiu e Strauch ${ }^{11}$ desenvolveram trabalho sobre o uso desse tipo de tubo em nervos ciáticos de cães, para lesões de até 10 milímetros de comprimento. Comprovaram o crescimento axonal por meio de estudos eletrofisiológicos e histológicos, demonstrando a efetividade do uso da técnica em relação ao crescimento e à maturação axonal. Trabalhos semelhantes foram realizados por Rice e Bernstein ${ }^{39}$, além de Wang e cols..$^{52}$, que confirmaram os resultados publicados anteriormente. Assim, esses estudos abriram caminho para o desenvolvimento de novas pesquisas experimentais sobre os tubos autólogos não-neurais.

Com o objetivo de aumentar a viabilidade dos tubos venosos, foram realizadas algumas pesquisas para avaliar a utilidade desses enxertos combinados a músculos esqueléticos. Essa combinação possibilitava o reforço da estrutura física da veia, evitando o seu colabamento. Alguns ensaios clínicos ${ }^{6,48}$ demonstraram bons resultados funcionais tanto em nervos sensoriais quanto em nervos mistos, para distâncias entre os cotos até mesmo maiores que 30 milímetros.

O uso de músculo esquelético autólogo para o reparo de nervos periféricos foi instituído em $1940^{15}$, mas em virtude de razões previamente expostas, a sua utilização difundiu-se apenas após a década de 1980. O uso desse material baseia-se nas similaridades histológicas entre a lâmina basal dos músculos estriados e a dos tubos endoneurais ${ }^{18}$. Esse tipo de enxerto geralmente é usado após pré-degeneração - com desnaturação de suas proteínas -, utilizando, para isso, calor ou frio. O procedimento visa a destruir os materiais histológicos com potencial teórico de dificultar a progressão axonal no interior do enxerto. Entretanto, estudos em animais realizados por Meek e cols. ${ }^{28}$, além de Migiliche e cols. ${ }^{29}$, não demonstraram diferença de resultados entre os músculos frescos e aqueles previamente desnaturados. Poucos estudos clínicos observaram bons resultados com o uso de enxertos autólogos de músculo esquelético, mesmo para o reparo de nervos ditos puramente sensitivos ${ }^{37}$.

Em 1989, Khouri e cols. ${ }^{23}$ realizaram um estudo com tubos venosos para tratamento de nervos ciáticos 
de ratos em lesões de até 10 milímetros. Os tubos foram enriquecidos com fatores de crescimento axonal (fatores de crescimento neuronal e de fibroblasto) e pequena camada de colágeno. Os resultados não demonstraram diferença significativa entre estes e os tubos ditos "vazios" (sem os fatores de crescimento).

Após o ressurgimento das experiências com o uso de tubos biológicos para o tratamento das lesões nervosas periféricas na década de 1980, alguns ensaios clínicos foram realizados, fornecendo as indicações para a utilização prática desses tecidos. Inicialmente, procurou-se analisar a efetividade dos tubos venosos quando comparados com os enxertos neurais autólogos em humanos, observando-se um padrão de regeneração axonal semelhante em casos de lesões de nervos sensitivos. Chiu e Strauch ${ }^{11}$ desenvolveram um estudo clínico comparando a regeneração funcional sensitiva entre tubos venosos, enxertos neurais autólogos e o reparo primário. Os resultados foram semelhantes quanto a falhas de tecido nervosos menores que 20 milímetros. Essas conclusões foram confirmadas em outros trabalhos, como o de Walton e cols. ${ }^{50}$, que relataram resultados semelhantes entre o uso de tubos venosos autólogos e o reparo direto de nervos em lesões de nervos digitais. Os autores observaram recuperação sensitiva razoável (grau S3+) segundo a escala de avaliação da British Medical Research Council.

Em 1993, Tang ${ }^{46}$ relatou o uso de tubos venosos enriquecidos com fragmentos de nervos autólogos em seu interior, para o tratamento de lesões dos nervos mediano e radial, em casos em que a perda de substância era maior que 45 milímetros. Os resultados obtidos com a técnica demonstraram boa recuperação motora (força M3 ou M4) em mais de $90 \%$ dos casos e recuperação de sensibilidade (S3 ou S4) em mais de 70\% dos casos. Outros estudos experimentais também têm comprovado a efetividade de tubos venosos combinados com músculo ou nervo. Os bons resultados devem-se, teoricamente, ao fato de esse tipo de combinação de tecidos proporcionar melhor suporte físico para o crescimento axonal (no caso de preenchimento com músculos), ou em razão da presença de uma fonte de células de Schwann, quando a veia é enriquecida com fragmentos de nervos ${ }^{2,3,47}$.

Embora muitos estudos experimentais confirmem a efetividade do uso de tubos venosos, a distância máxima em que seu uso pode ser sugerido ainda é motivo de controvérsia. Na tentativa de responder a essa questão, Strautch ${ }^{44}$ realizou um estudo em nervo fibular de ratos, comparando a taxa histológica de crescimento axonal em tubos venosos usados em diferentes distâncias de falha neural - variação entre 10 e 60 milímetros. Esse estudo confirmou as observações clínicas e empíricas de que o uso de tubos venosos puros só seria efetivo em distâncias menores que 30 milímetros. Reforçou também as teorias de que, para o tratamento de lesões mais extensas, os tubos venosos autólogos deveriam ser acrescidos de outros materiais, como músculos ou nervos, ou enriquecidos com substâncias que incentivassem o crescimento axonal, como fatores de crescimento.

\section{Tubos artificiais}

Como descrito previamente, as técnicas de tubulização apresentaram grande desenvolvimento nas últimas duas décadas, chamando a atenção dos pesquisadores por terem se mostrado como alternativas viáveis para o tratamento das lesões do sistema nervoso periférico. As pesquisas com o uso de tubos artificiais iniciaramse logo após o ressurgimento do interesse por tubos biológicos não-neurais e uma série de diferentes tipos de materiais sintéticos já foi testada, tais como metais inertes, celulose, gelatina ou plástico ${ }^{36}$. O primeiro relato do uso de um tubo sintético deve-se a Garrity ${ }^{17}$, que descreveu a utilização ineficaz de polietileno em três pacientes com lesões de nervo periférico com considerável intervalo entre os cotos. Os resultados obtidos com o uso de tântalo para o reparo de lesões nervosas em soldados da Segunda Guerra Mundial foram também desapontadores. Assim, como já havia ocorrido com relação ao uso de tubos biológicos, a tubulização com uso de enxertos artificiais foi um procedimento praticamente esquecido nas décadas de 1960 e $1970^{14}$.

Nas duas décadas que se seguiram, renasceu o interesse pela técnica, objetivando principalmente a obtenção de uma forma de reparo que não provocasse uma "segunda agressão" ao organismo ${ }^{33}$. Os primeiros estudos envolveram materiais não absorvíveis. Alguns desses materiais, como os condutos de polietileno, apresentaram resultados conflitantes em estudos experimentais: Stanec e $\operatorname{Stanec}^{42}$ realizaram um estudo experimental demonstrando bons resultados em termos de recuperação funcional; resultados esses não comprovados por Pitta e cols. ${ }^{38}$

O uso de silicone como opção de reparo foi descrito inicialmente há mais de 20 anos, por Dahlin e Lundborg $^{12}$. Em estudo experimental em ratos, esses autores puderam observar o crescimento axonal proximal em tubos usados para o reparo de nervos em que não havia perda de substância (falhas menores que 5 milímetros), com resultados comparáveis ao reparo primário. Esses resultados foram reproduzidos por outros pesquisadores que, além de comprovar a eficácia da técnica, também demonstraram a presença de grande quantidade de substâncias que estimulam o crescimento axonal (como fator 
de crescimento de axônios e fibroblastos) no interior desses tubos de silicone. Assim, comprovou-se a síntese desses produtos a partir de células de Schwann dos axônios do coto distal, que são liberados dentro do tubo, estimulando o crescimento axonal. Do ponto de vista histológico, pesquisas experimentais demonstraram que ocorre formação de matriz de fibrina dentro dos tubos de silicone, o qual é posteriormente colonizado por macrófagos, fibroblastos e células de Schwann.

Aliados à busca de tubos artificiais não absorvíveis, pesquisadores também iniciaram a análise de materiais absorvíveis para uso como tubo neural. A vantagem desses materiais deve-se ao fato de que, teoricamente, não induziriam à reação do tipo corpo estranho. Dessa forma, poder-se-ia evitar o desencadeamento de processo inflamatório local e conseqüente fibrose, eventos que acabam por dificultar a regeneração axonal. O colágeno é um dos enxertos bioabsorvíveis que mais foi testado em trabalhos experimentais. Vários autores já descreveram seu uso para o reparo de lesões de nervos periféricos em animais, obtendo conclusões variáveis. Madorsky e cols. ${ }^{27}$ observaram resultados comparáveis entre sutura direta e o uso de tubos de colágeno para o reparo de nervos sensitivos. Hitaha e cols..$^{21}$ utilizaram colágeno para regeneração de nervos faciais lesados em gatos, obtendo boa recuperação funcional. Heike e cols. ${ }^{20}$ demonstraram resultados semelhantes no reparo de nervos sensoriais de coelhos, comparando a sutura direta em relação ao tubo de colágeno de suíno processado, nas lesões com falhas de até 10 milímetros. Além do colágeno, também o ácido poliglicólico (PGA) foi exaustivamente avaliado em estudos experimentais. Nakamura e cols. ${ }^{34}$ observaram que os tubos desse material demonstraram melhores resultados funcionais do que os enxertos autólogos para o tratamento de falhas de 15 milímetros em nervos ciáticos de cães. Bini e cols. ${ }^{4}$ identificaram $90 \%$ de regeneração axonal em lesões de nervo ciático de ratos com o uso de tubos finos de PGA. Wang e cols. ${ }^{52}$ associaram PGA a um conduto de Chitosan (que é um tipo de material microporoso, polissacarídeo derivado do exoesqueleto de crustáceos) e observaram recuperação em todos os cães com lesão de nervo ciático com intervalo de 30 milímetros. Mais recentemente, um novo polímero foi testado em ratos, para lesões de nervo mediano com intervalo de 20 milímetros, o trimetileno-co-épsilon-caprolactona (TMC/CL), o qual demonstrou resultados satisfatórios somente quando usado em associação a culturas de células de Schwann ${ }^{41}$.

Com o passar dos anos, vários modelos experimentais foram testados no processo de tubulização. Entretanto, apenas o silicone e os tubos absorvíveis de PGA foram avaliados em ensaios clínicos. A maior experiência no uso dos condutos de silicone em humanos pertence ao grupo de Lundborg e cols. ${ }^{25}$, que, em
2004, publicou importante ensaio clínico, prospectivo e randomizado, do uso do silicone em reparos de lesões cortantes dos nervos ulnar e mediano, na região distal do antebraço. Nesse estudo, considerando um acompanhamento de cinco anos, os autores compararam os resultados do reparo primário microcirúrgico convencional ao resultado com uso de tubos de silicone em falhas de até 5 milímetros. Utilizando os parâmetros clínicos definidos pelo British Medical Research Council e a Escala Sensorial de Dellon para a avaliação dos resultados cirúrgicos, observou-se que, nos primeiros três meses de seguimento, houve diferença da recuperação funcional em favor da tubulização, mas essa diferença tornou-se inexistente após um ano de seguimento. Além disso, após cinco anos, não se demonstrou diferença com relação às recuperações funcionais sensitiva e motora entre as duas técnicas operatórias. Cabe ressaltar que foi observada apenas melhor tolerância ao frio nos casos em que foi realizada tubulização. Com relação à fibrose, possivelmente induzida por materiais inertes como o silicone, foi necessária a retirada do tubo em apenas 7 casos, em um grupo de 30 participantes, em razão de queixas de desconforto local mecânico.

Já foram publicados alguns estudos demonstrando a efetividade dos tubos de PGA para o reparo de lesões nervosas periféricas ${ }^{26}$ em humanos. Em 1999, esse tipo de tubo condutor foi liberado pela FDA (Food and Drug Administration) para uso em humanos nos Estados Unidos, sob rótulo comercial de Neurotube ${ }^{\circledR}$ (Synovis Microsystems, Birmingham, AL). Em 2000, foi publicado um estudo multicêntrico, randomizado e prospectivo, que avaliou o uso desse tipo de tubo para reconstrução de nervos digitais, envolvendo 96 pacientes $^{53}$. O estudo demonstrou que, naqueles casos em que os condutos foram utilizados, se obtiveram melhores resultados nas pequenas falhas (menores que 4 milímetros) quando comparados com suturas término-terminais. Além disso, também demonstrou resultados melhores, em termos de recuperação sensitiva, quando os tubos eram usados para falhas maiores que 30 milímetros, se comparados com os reparos feitos com enxertos de nervos autólogos. Os condutos de PGA também foram utilizados para o reparo de alguns outros nervos, mas todas as publicações apresentam número muito limitado de pacientes, sendo a maioria restrita a relato de casos. O grupo de Dellon reportou seu uso em dois casos, especificamente lesão dos nervos plantar medial ${ }^{24}$ e acessório ${ }^{13}$, obtendo excelente recuperação funcional. Inada e cols. ${ }^{22}$ observaram recuperação sensitiva completa com o uso desses tubos no reparo de um nervo fibular superficial e Navissano e cols. ${ }^{35}$ utilizaram-se dessa técnica para o reparo em sete pacientes com lesões distais do nervo facial, identificando $71 \%$ de resultados considerados positivos pelos autores. 


\section{Técnica cirúrgica e indicações atuais para uso na prática clínica}

A técnica cirúrgica mais empregada para a confecção da tubulização resume-se na introdução dos cotos do nervo lesado no interior de tubos previamente preparados. Os nervos são ancorados ao tubo através de duas a quatro suturas, utilizando-se fios de náilon ou prolene 9-0 (figura 1). Deve-se sempre procurar manter os nervos sem torção, para se evitar alterações na orientação de seus fascículos. O uso de cola biológica é uma alternativa que reduz a manipulação dos cotos. A técnica envolve variações de acordo com o tipo de tubo utilizado ${ }^{2,35}$. Os condutos podem ser sintéticos ou retirados a partir de tecidos adjacentes, contendo ou não substâncias em seu interior, como fatores de crescimento neural, Matrigel ${ }^{\circledR}$, matriz de colágeno ${ }^{51}$, ou culturas de células de Schwann ${ }^{41}$. Os tubos provenientes de material autólogo requerem maior tempo para o seu preparo, pois há necessidade de ressecção e preparação de veias e/ou músculos esqueléticos. Esses tubos, em geral, são obtidos a partir de tecidos do próprio leito cirúrgico. No caso de veias, deve-se procurar obter vasos que apresentem diâmetro o mais próximo possível daquele do nervo lesado. Deve-se também tomar o cuidado de ligar adequadamente a emergência de pequenas veias colaterais, para evitar uma posterior dispersão axonal ${ }^{6}$.

Os estudos da literatura apontam que resultados funcionalmente satisfatórios podem ser obtidos tanto com o uso de materiais biológicos quanto com condutores sintéticos. Atualmente, a tubulização já se apresenta, em situações clínicas selecionadas, como uma alternativa viável ao uso de enxertos de nervos autólogos. Os dados clínicos sugerem que os tecidos biológicos compostos de veias ou músculos esqueléticos têm boa possibilidade de sucesso quando utilizados para o reparo de lesões com intervalos pequenos entre os cotos (até 30 milímetros). Tal fato também se aplica aos tubos de silicone, considerando uma distância limite de no máximo 25 milímetros. Em lesões cujas distâncias entre os cotos são longas (maiores que 30 milímetros), os enxertos biológicos combinados, como veia e músculo ou veia e nervos, podem ser utilizados como alternativa terapêutica.

Os tubos bioabsorvíveis de PGA parecem representar uma opção razoável para situações clínicas seletas, em especial nos casos de lesões de nervo digital. Porém, até o momento não foram desenvolvidos estudos abrangendo grandes séries a respeito do uso desses tubos em lesões de nervos mistos. Assim, atualmente ainda não é possível a recomendação de seu uso como alternativa aos enxertos neurais autólogos.

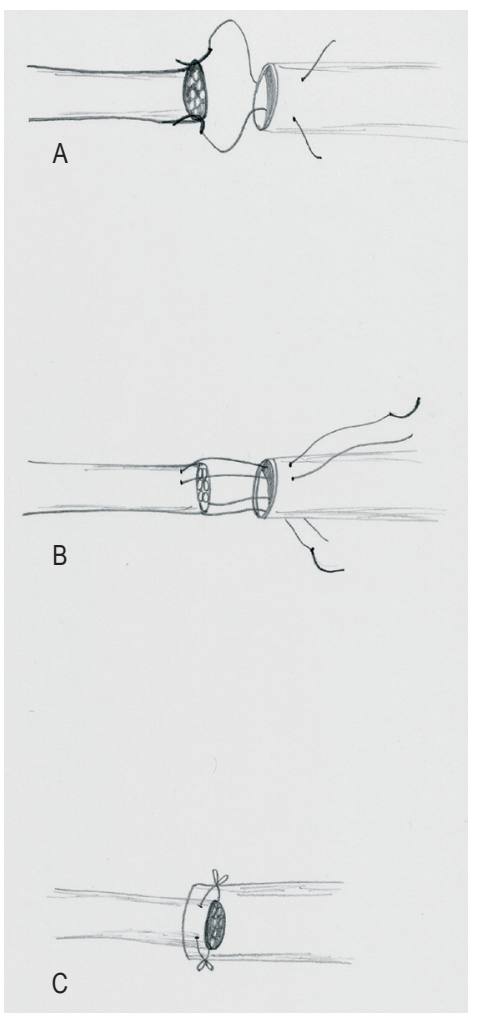

Figura 1 - Técnica cirúrgica empregada. A) passagem do fio pelo tubo no sentido de fora para dentro, sendo então transpassado no epineuro do coto nervoso, $0,5 \mathrm{~cm}$ proximalmente ao local de secção do nervo; $B)$ o fio volta a transpassar o tubo no sentido de dentro para fora; $C$ ) confecção do ponto de sutura, dessa forma inserindo a ponta do coto nervoso no interior do tubo.

\section{Conclusão}

Atualmente, a maioria dos autores concorda que os enxertos neurais autólogos são a primeira escolha para o tratamento das lesões de nervos periféricos nas situações em que a sutura primária dos cotos não é possível. Entretanto, os estudos experimentais em animais e alguns ensaios clínicos em humanos sugerem que os tubos biológicos ou sintéticos podem funcionar como opção aos enxertos neurais em situações selecionadas. $\mathrm{O}$ ressurgimento do interesse sobre esse tema nas últimas duas décadas proporcionou a geração de informações que detalharam os mecanismos básicos e fisiopatológicos que regem esse tipo de técnica. Essas informações poderão guiar futuras pesquisas que tenham como objetivo o desenvolvimento de condutores alternativos, viabilizando a substituição dos enxertos autólogos nas cirurgias do sistema nervoso periférico. 


\section{Referências}

1. BALANCE C, DUELAB: Operative treatment of facial palsy by the introduction of nerve grafts. Arch Otol 15:1, 1932.

2. BATTISTON B, TOS P, CUSHWAY T, GEUNA S: Nerve repair by means of vein filled with muscle grafts: clinical results. Microsurgery 20:32-6, 2000.

3. BATTISTON B, TOS P, GEUNAS, GIACOBINI-ROBECCHI MG, GUGLIEUMONE R: Nerve repair by means of vein filled with muscle grafts: morphological analysis of regeneration. Microsurgery 20:37-41, 2000.

4. BINI TB, GAO S, XU X, WANG S, LEONG KW: Peripheral nerve regeneration by mycrobraided poly (L-lactide-co-glycolide) biodegradable polymer fibers. J Biomed Matter Res 1: 286-95, 2004.

5. BROOKS D, TERZIS JK: The place of nerve grafting in orthopaedic surgery. J Bone Joint Surg 37A: 299, 1955.

6. BRUNELLI G, BATTISTON B, VIGASIO A: Brinding veins defects with combined skeletal muscle and vein conduits. Microsurgery 14:247-51, 1993.

7. BUNELL S: Surgery of nerves of the hand. Surg Gynecol 44:145, 1927.

8. BUNELL S, BOYES H: Nerve grafts. Am J Surg 45:54, 1939.

9. BUNGNER OV: Ueber die degenerations und regenerations - Vorgange am nerven nach Verletzungen. Beitr Pathol Anal 10:321, 1891.

10. CHIU DTW: Autogenous vein graft as a conduit for nerve regeneration. Surg Forum 31:550-55, 1980.

11. CHIU DTW, STRAUCH B: A prospective clinical evaluation of autogenous vein grafts used as nerve conduit for distal sensory nerve defects of $3 \mathrm{~cm}$ or less. Plast Reconstr Surg 86:928-34, 1990.

12. DAHLIN LB, LUNDBORG G: Use of tubes in peripheral nerve repairs. Neurosurg Clinic North Am 12:341-52, 2001.

13. DUCIC J, MALONEY CT Jr, DELLON AL: Reconstruction of the spinal acessory nerve with autograft or neurotube? Two case reports. J Reconstruct Microsurg 21: 29-33, 2005.

14. DUCKER TB, HAYES GJ: Experimental improvements in the use of Silastic cuff for peripheral nerve repair. J Neurosurg 28:582-87, 1968.

15. FAWCETT JW, KEYNES RJ: Muscle basal lamina: a new graft material for peripheral nerve repair. J Neurosurg 65:354-63, 1986.

16. FORAMETTI C: Zur technik der nervennaht. Arch Klin Chir 73:643, 1904

17. GARRITY RW: The use of plastic and rubber tubing in the management of irreparable nerve injuries. Surg Forum 6:517-20, 1995

18. GLASBY MA, GSCHMEISSNER SG, HITCHCOCK RJI: A comparison of nerve regeneration trough nerve and muscle grafts in rat sciatic nerve. Neurorthopaedics 2:21-28, 1986.

19. GLUCK T: Ueber neuroplastic Auf dem Wege dem Transplantation. Arch Klin Chir 25:606-16, 1880.

20. HEIKE CM, KLOPPER J, BALJET B: Processed porcine collagen tubulization versus conventional suturing in peripheral nerve reconstruction: an experimental study in rabbits. Microsurgery 21:84-95, 2001.

21. HITAHA AK, NISHIMURA Y, SHIMIZU Y, ENDO K: Facial nerve repair accoplished by the interposition of a collagen nerve guide. J Neurosurg 93:113-20, 2000.

22. INADA Y, MORIMOTO S, TAKAKURA Y, NAKAMURA T: Regeneration of peripheral nerve gap with a polyglicolic acid-collagen tube. Neurosurgery 55: 640-6, 2004.
23. KHOURI RK, CHIU DTW, FEINBERG J: Effects of neurite-promoting factors on rat sciatic nerve regeneration. Microsurgery 10:206-9, 1989.

24. KIM J, DELLONAL: Reconstruction of a painful post-traumatic medial plantar neuroma with a bioabsorbable nerve conduit: a case report. J Foot Ankle Surg 40: 318-23, 2001.

25. LUNDBORG G, ROSEN B, DAHLIN L, HOMBERG J, ROSE N: Tubular repair of the median or ulnar nerve in the human forearm: a 5 year follow-up. J Hand Surg (Br) 29:100-7, 2004.

26. MACKINNON SE, DELLONAL: Clinical nerve reconstruction with bioabsorbable polyglycolic acid tube. Plast Reconstr Surg 85:419-24, 1990.

27. MADORSKY SJ, SWETT JE, CRUMLEY RL: Motor versus sensory neuron regeneration trough collagen tubulus. Plat Reconstr Surg 103:430-8, 1998.

28. MEEK MF, DEN DUNNEN WFA, SCHAKENRAAD JM, ROBINSON PH: Evaluation of several techniques to modify denatured muscle tissue to obtain a scaffold for peripheral nerve regeneration. Biomaterials 20:101-8, 1999.

29. MIGILICHE N, TABATA Y, ENDOH K, IDE C: Peripheral nerve regeneration trough a long detergent-denatured muscle autografts in rabbits. Neuroreport 12:1719-22, 2001.

30. MILLESI H: Interfascicular nerve grafting. Orthop Clin North Am 2:419-35, 1970.

31. MILLESI H, MEISSL G, BERGER A: The interfascicular nerve-grafting of the median and ulnar nerves. J. Bone Joint Surg (Am) 54:722-50, 1972.

32. MILLESI H, MEISSL G, BERGER A: Further experience with interfascicular grafting of the median, ulnar and radial nerves. J Bone Joint Surg (Am) 58:209-18, 1976.

33. NAFF NJ, ECKLUND JM: History of peripheral nerve surgery techniques. Neurosurg Clin North Am 12:197-209, 2001.

34. NAKAMURA T, INADA Y, FUKUDA S, YASHITANI $M$ et al: Experimental study on the regeneration of peripheral nerve gaps through a poliglycolyc acid (PGA-collagen) tube. Brain Res 1027:18-29, 2004.

35. NAVISSANO M, MALAN F, CARNINO R, BATTISTON B: Neurotube for facial nerve repair. Microsurgery 25:268-71, 2005.

36. PAYR E: Beitrage zur technik der Blutgefass und Nervennaht nebst Mitteilungen uber die Verwendung eines resorbibaren Metalles in der Chirurgie. Arch Klin Chir 62:67-111, 1900.

37. PEREIRA JH, BOWDEN REM, GATTUSO JM, NORRIS RW: Comparison of results of repair of digital nerves by denatured muscle grafts and end-to-end sutures. J Hand Surg (Br) 16:519-523, 1991.

38. PITTA MC, WOLDFORD LM, MEHRA P, HOPKIN J: Use of Gore-Tex tubing as a conduit for inferior alveolar and lingual nerve repair: experience with 6 cases. J Oral Maxillofac Surg 59:493-6, 2001.

39. RICE DH, BERNSTEIN FD: The use of autogenous vein for nerve grafting. Otolaryngol Head Neck Surg 92:410-6, 1984.

40. SEDDON S: Surgical disorders of the peripheral nerves. Edinburgh, Churchil-Livisgstone, 1972.

41. SINIS N, SCHALLER HE, SCHULTE-EVERSUM C, SHLOSSHAUER B, DOSER M et al: Nerve regeneration across a 2-cm gap in the rat median nerve using a resorbable nerve conduit filled with Schwann cells. J Neurosurg 103:1067-76, 2005.

42. STANEC S, STANEC Z: Reconstruction of upper-extremity peripheral nerve injuries with ePTFE conduits. J Reconstr Microsurg 14:227-32, 1988.

43. STRANGE FGC: An operation for nerve pedicule graft. Preliminary communication. Br J Surg 34:423, 1947. 
44. STRAUTCH B: Determining the maximal length of a vein conduit used as an interposition graft for nerve regeneration. J Reconstr Microsurg 12:521-7, 1996.

45. SUDERLAND S: Nerve injuries. 2.ed. New York, Churchill Livingston, 1978.

46. TANG JB: Repair of digital nerve with autogenous vein graft during flexor tendon surgery in zone 2. J Hand Surg $(\mathrm{Br})$ 18:449-53, 1993.

47. TOS P, BATTISTON B, GEUNA S, GIACOBIN-ROBECCHI MG, HILL MA, LANZETTA M et al.: Tissue specifity in rat peripheral nerve regeneration trough combined skeletal muscle and vein conduit grafts. Microsurgery 20:65-71, 2000.

48. TOS P, CALCAGNI M, GIGO-BENATO D: Use of muscle-vein combined Y-chambers for repair of multiple nerve lesions: experimental results. Microsurgery 24:459-64, 2004.

49. VANLAIR C: Nouvelles recherches expèrimentales sur la régénération dês nerfs. Arch Biol Paris 12:235, 1885.

50. WALTON RL, BROWN RE, MATORY WE: Autogenous vein graft repair of digital nerve defects in the finger: A retrospective clinical study. Plast Reconstr Surg 84:944, 1989.

51. WANG KK, COSTAS PD, JONES DS: Sleeve insertion and collagen coating improve nerve regeneration trough vein conduits. J Reconstr Microsurg 9:39-48, 1993.

52. WANG X, HU W, CAO Y, YAO J, WU J, GU X: Dog sciatic nerve regeneration across a $30-\mathrm{mm}$ defect brigded by a chitosan/PGA artificial nerve graft. Brain 128: 1897-910, 2005.

53. WEBER RA, BREIDENBACH WC, BROWN RE, JABALEY ME, MASS DP: A randomized prospective study of polyglycolic acid conduits for digital nerve reconstruction in humans. Plast Reconstr Surg 106:1036-45, 2000.

54. WEISS P: Experiments on nerve repair. Tram Neurol Arch 69:42, 1943

55. WREDE L: Uberbrueckung eines Nervendefektes mittels Seidennaht und leben venenstueckes. Dtsch Med Wochenschr 35:1125-60, 1909.

Original recebido em abril de 2006

Aceito para publicação em dezembro de 2006

Endereço para correspondência

Leandro Pretto Flores

SQN 208, bloco F, ap. 604

70853-060 - Brasilia, DF

E-mail: leandroprettoflores@hotmail.com

\section{Comentários}

Os avanços da bioengenharia permitiram, nos últimos anos, o desenvolvimento de tubos sintéticos absorvíveis, em especial os de ácido poliglicólico e de colágeno, que ampliaram as possibilidades de reparo das lesões de nervos periféricos. Os autores realizaram uma revisão detalhada sobre o tema e a apresentam de forma ordenada e de fácil leitura.

Colaborando para a exatidão do trabalho, seria importante acrescentar que, além dos estudos clínicos sobre os tubos de ácido poliglicólico descritos no artigo, foram publicados recentemente estudos clínicos também com os tubos de colágeno (Neuragen ${ }^{\circledR}$, Integra Neurosciences, Plainsboro, NJ), relatando bons resultados ${ }^{1,2}$.

No momento, não existem evidências clínicas convincentes de que os reparos com tubos condutores levem a um resultado funcional melhor que os dos enxertos autólogos, embora em algumas situações (mencionadas no artigo) resultados pelo menos comparáveis possam ser alcançados. É também importante lembrar que, embora esses tubos pareçam ideais, sua reabsorção pode trazer problemas adicionais em razão da invasão macrofágica, fibrose e crescimento axonal desordenado. Além disso, os resultados positivos relatados na literatura são, na maioria dos casos, no reparo de defeitos com até 20 milímetros de extensão.

Apesar dessas ressalvas, as possibilidades de manipulação estrutural por métodos de engenharia tecidual, associadas à possível inclusão de células de Schwann, fatores neurotróficos e outros agentes estimuladores da regeneração neural, farão que, no futuro, os tubos condutores sintéticos absorvíveis ocupem posição importante entre as técnicas de reparo de nervos periféricos.

Gostaria de congratular os autores por mais essa significativa contribuição à literatura relacionada ao tratamento cirúrgico das lesões de nervos periféricos.

\author{
Mário G. Siqueira \\ Grupo de Cirurgia de Nervos Periféricos da \\ Divisão de Neurocirurgia do Hospital das Clínicas da \\ Faculdade de Medicina da Universidade de São Paulo
}

\section{Referências}

1. TARAS JS, NANAVATI V, STEELMAN P: Nerve conduits. J Hand Ther 18:191-7, 2005.

2. TRUMBLE TE, PARISI D, ARCHIBALD S, ALLAN CH: Synthetic nerve conduits. In: SLUTSKY DJ, HENTZ VR (eds.): Peripheral nerve surgery: practical applications in the upper extremity. Philadelphia, Churchill Livingstone, 2006, pp 121-8. 